\title{
Analyzing stability of equilibrium points in impulsive neural network models involving generalized piecewise alternately advanced and retarded argument
}

\author{
Kuo-Shou Chiu ${ }^{1}$ \\ ${ }^{1}$ Universidad Metropolitana de Ciencias de la Educación
}

February 6, 2021

\begin{abstract}
In this paper, we investigate the models of the impulsive cellular neural network with piecewise alternately advanced and retarded argument of generalized argument (in short IDEPCAG). To ensure the existence, uniqueness and global exponential stability of the equilibrium state, several new sufficient conditions are obtained, which extend the results of the previous literature. The method is based on utilizing Banach's fixed point theorem and a new IDEPCAG's Gronwall inequality. The criteria given are easy to check and when the impulsive effects do not affect, the results can be extracted from those of the non-impulsive systems. Typical numerical simulation examples are used to show the validity and effectiveness of proposed results. We end the article with a brief conclusion.
\end{abstract}

\section{Hosted file}

wileyNJD-AMA.pdf available at https://authorea.com/users/394160/articles/507610analyzing-stability-of-equilibrium-points-in-impulsive-neural-network-models-involvinggeneralized-piecewise-alternately-advanced-and-retarded-argument 